\title{
Anomalous nighttime electron temperatures over Millstone Hill: a statistical study
}

\author{
V. V. Lobzin and A. V. Pavlov \\ Institute of Terrestrial Magnetism, Ionosphere, and Radio-Wave Propagation, Russian Academy of Sciences (IZMIRAN), \\ Troitsk, Moscow Region, 142190, Russia
}

Received: 17 December 2002 - Revised: 1 June 2003 - Accepted: 18 June 2003 - Published: 1 January 2004

\begin{abstract}
A statistical study of anomalous nighttime electron temperature enhancements, NETEs, observed on 336 nights during Millstone Hill radar measurements on 730 nights from 1976 to 2001 is carried out. NETEs are most frequent in winter and in autumn. The NETE occurrence has a maximum probability in February and a minimum probability in July. The asymmetry between spring and autumn NETE occurrences is found for NETEs, which are observed during geomagnetially quiet time periods. The calculated value of the NETE occurrence probability is decreased with the solar activity index F10.7 increase. The increase in a 3-h geomagnetic index $K_{p}$ or the decrease in a 1-h geomagnetic index $D_{s t}$ leads to the increase in the NETE occurrence probability. This tendency is more pronounced for current values of $K_{p}$ or $D_{s t}$ rather than for delayed ones and becomes more weak with the delay increase. The NETEs are most likely to begin between 19:00 and 20:00 UT. The studied NETEs are characterized by the most typical duration from 1 to $3 \mathrm{~h}$ with the percentage peak between 1 and $2 \mathrm{~h}$. The electron temperature increases are predominately between $100 \mathrm{~K}$ and $300 \mathrm{~K}$. We did not find any relationship between the amplitude and duration of the NETEs studied. It is shown that there is a tendency for the NETE amplitude to increase if the value of $K_{p}$ or $\left|D_{s t}\right|$ increases. To determine whether there exists a difference between NETEs observed during different solar cycles, we chose the data subsets corresponding to 21 and 22 solar cycles and performed the statistical studies for each subset. It was found that, within the errors, the corresponding dependencies are the same for the cycles considered and for the entire data set.
\end{abstract}

Key words. Ionosphere (plasma temperature and density; ionospheric disturbances; modeling and forecasting)

\section{Introduction}

Unusually high nighttime F-region electron temperatures over Millstone Hill were first observed in 1964 by Evans (Evans, 1967), and the measurements of these Millstone Hill

Correspondence to: V. V. Lobzin (lobzin@izmiran.rssi.ru) anomalous electron temperatures were presented in many subsequent papers (e.g. Evans and Holt, 1978; Babcock and Evans, 1979; Garner et al., 1994; Pavlov, 1994; Richards and Khazanov, 1997; Pavlov et al., 1999, 2000, 2001; Pavlov and Oyama, 2000; Richards et al., 2000; Pavlov and Foster, 2001). Anomalous high electron temperatures in the mid-latitude winter nighttime ionosphere were also found by Sanatani and Breig (1981) using OGO 6 data. Garner et al. (1994) used the data on 173 nights between 1967 and 1974 and found that nighttime electron temperature enhancements, NETEs, were observed during 41 nights. Using this limited data set, Garner et al. (1994) carried out a statistical study of these NETEs and examined the occurrence frequency, the duration, the time of onset, the magnitudes of temperature increases, and the dependence on solar and geomagnetic activity. For some of the conclusions deduced by Garner et al. (1994), a statistical validity is unclear due to the very limited data set used. The main aim of the present paper is to carry out a more reliable statistical study of NETEs with the use of Millstone Hill radar data on 730 nights from 1976 to 2001 . The results obtained are compared with early results of Garner et al. (1994) to emphasise the newness of our results.

An anomalous nighttime electron density enhancement, NEDE, is also a well-known middle latitude F-region phenomenon (e.g. Evans, 1965, 1974; Da Rosa and Smith, 1967; Rao et al., 1982). In our analysis we study the relationships between NETEs and the changes in the electron density during these events. It makes clear that there exists a possibility to observe a NEDE during a NETE.

\section{Data and method of data analysis}

Ionospheric radar observations from Millstone Hill $\left(42.6^{\circ} \mathrm{N}\right.$, $288.5^{\circ} \mathrm{E}$ ) taken on 730 nights with an overall duration of $6372 \mathrm{~h}$ from 2 February 1976 to 10 September 2001 are the subject of the present study. We use the $300-\mu$ s pulse length data which have an altitude resolution of $45 \mathrm{~km}$. NETEs were observed on 336 nights during the studied time period. 


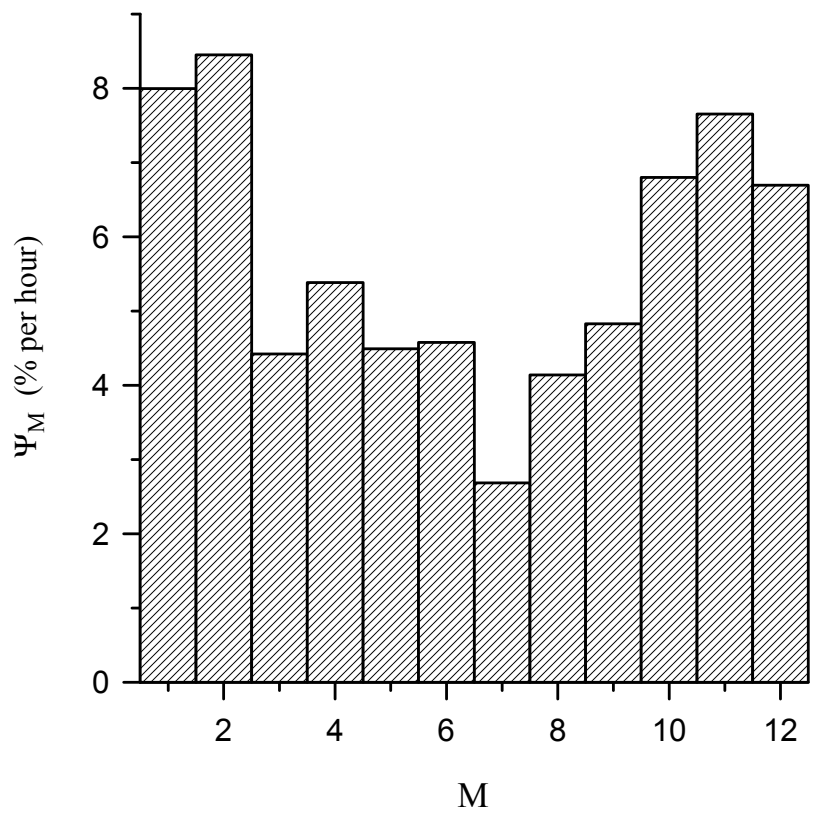

Fig. 1. The dependence of the NETE occurrence probability $\Psi_{M}$ on a number $M$ of a given month in a year.

Our definition of a NETE is close to that given by Garner et al. (1994). Data are averaged over half-hour time periods to decrease effects of errors of measurements of electron temperatures, $T$, and electron number densities, $N$. Like Garner et al. (1994), the data are interpolated to obtain the values at $400 \mathrm{~km}$. We give a nighttime electron temperature enhancement the labels "NETE" if the electron temperature increase is greater than $100 \mathrm{~K}$, i.e. when the temperature enhancement exceeds any experimental uncertainties (Garner et al., 1994). In order to avoid sunrise or sunset effects, we analyse only observations for large enough zenith angles, $\chi \geq 100^{\circ}$.

A NETE event begins, when a relative minimum in electron temperature is observed. The peak electron temperature is the relative maximum. Like Garner et al. (1994), the assumed NETE duration is the length of time interval between the onset of the event and the peak, and events with duration less than 1 hour are not taken into consideration. The amplitude of a NETE is defined as the difference between the peak temperature and the onset temperature.

We analyze 392 NETE events which were observed during 336 nights. Single NETE events per night were observed by the Millstone Hill radar during 286 nights, two NETEs per night were found during 44 nights, and three NETEs per night were observed during 6 nights.

We study the dependence of the probabilities of NETE occurrences during 1-h nighttime periods on a daily solar activity index, F10.7, a 3-h geomagnetic index, $K_{p}$, a 1-h geomagnetic index, $D_{s t}$, a number, $M$, of a given month in a year, and an onset local time, $t_{0}$. Let $X$ be some of the parameters mentioned. The corresponding probability function, $\Psi_{X}$, is estimated as a ratio of a number of NETE observations that occur for a given interval of $X$ to the total duration of nighttime measurements for the same interval of $X$. It should be noted that the duration of radar measurements has noticeable seasonal changes for the analyzed data set, and the simple percentage may have no physical importance. Analogously, the duration of observations for different intervals of solar and geomagnetic indices are not equal to each other, and it is this fact that generates the need to use the probability per hour of observations to reveal physical causes of the events considered. To estimate the errors for the occurrence probabilities, we assume that for given conditions the probability distribution function for the number of NETE events, $N_{\mathrm{NETE}}$, can be considered as Gaussian with the variance $\sigma^{2} \approx N_{\text {NETE}}$. In this case, we can use $\sigma$ as a measure of statistical fluctuations of the number of events observed. It should be noted that approximately $68 \%$ of all observations should fall within a range of $\pm \sigma$ from the mean; the fluctuations with larger amplitudes have a relative frequency of $32 \%$ or less.

\section{Results and discussion}

\subsection{Seasonal variations in the NETE occurrence probabil- ity}

Figure 1 shows the dependence of the NETE occurrence probability $\Psi_{M}$ on a number of a given month in a year. It can be seen from Fig. 1 that the NETE occurrence probability is the lowest in July. However, in contrast to the conclusions of Garner et al. (1994) about the almost complete disappearance of NETEs in summer, the values of $\Psi_{M}$ found in the autumn and spring months are close to that in the summer months. One can notice from Fig. 1 that the maximum value of the NETEs occurs in February, and this result is in general agreement with more early conclusions of Garner et al. (1994) about the predominance of NETE occurrences in winter.

The 3-month averages of $\Psi_{M}$, presented in Table 1, show the seasonal distribution of all studied NETE observations. As usual, we assume that December, January, and February are the winter months; March, April, and May correspond to spring; June, July, and August are the summer months; and September, October, and November correspond to autumn. Relative to the summer magnitude of $\Psi_{M}$, the other average seasonal values of $\Psi_{M}$ give an increase with the maximum probability in winter. The asymmetry between the spring and autumn months is seen in Table 1 for the NETE occurrence probability, with a stronger autumn enhancement. However, it will be shown in Sect. 3.3 that the NETE occurrence probability increases with the geomagnetic activity $K_{p}$ index. This dependence could form a part of the seasonal dependence of $\Psi_{M}$ shown in Fig. 1 and in Table 1 for all events, if the studied events are distributed non-uniformly between the seasons. To examine this hypothesis, we divide the measurements into two groups corresponding approximately to the geomagnetically quiet and disturbed conditions. When the thermosphere is disturbed, it takes time for it to relax back 
Table 1. Average values of $\Psi_{M}$ (\% per hour) during winter, summer, spring, and autumn months for all observations, as well as for measurements made under geomagnetically quiet and disturbed conditions. The conditions are considered to be quiet if current $K_{p} \leq 3$ (I), $K_{p} \leq 3$ during $12 \mathrm{~h}$ (II) or $24 \mathrm{~h}$ (III) before the measurement, otherwise the conditions are disturbed.

\begin{tabular}{|c|c|c|c|c|c|c|c|}
\hline & \multirow{2}{*}{$\frac{\text { all }}{\text { events }}$} & \multicolumn{2}{|c|}{ I } & \multicolumn{2}{|c|}{ II } & \multicolumn{2}{|c|}{ III } \\
\hline & & quiet & disturbed & quiet & disturbed & quiet & disturbed \\
\hline Winter & $7.8 \pm 0.6$ & $7.1 \pm 0.7$ & $9.1 \pm 1.2$ & $7.3 \pm 0.9$ & $8.2 \pm 0.9$ & $7.1 \pm 1.0$ & $8.2 \pm 0.9$ \\
\hline Spring & $4.8 \pm 0.6$ & $1.8 \pm 0.4$ & $10.1 \pm 1.4$ & $1.5 \pm 0.5$ & $7.7 \pm 1.0$ & $1.2 \pm 0.5$ & $6.6 \pm 0.8$ \\
\hline Summer & $3.8 \pm 0.7$ & $3.1 \pm 0.6$ & $7.5 \pm 2.3$ & $2.4 \pm 0.6$ & $6.9 \pm 1.6$ & $2.4 \pm 0.7$ & $5.3 \pm 1.1$ \\
\hline Autumn & $6.7 \pm 0.6$ & $5.3 \pm 0.6$ & $9.5 \pm 1.2$ & $4.8 \pm 0.7$ & $8.4 \pm 0.9$ & $5.9 \pm 0.9$ & $7.1 \pm 0.7$ \\
\hline
\end{tabular}

to its initial state, and this thermosphere relaxation time determines the time for the disturbed ionosphere to relax back to the quiet state. It means that not every time period with sufficiently low $K_{p}$ (e.g. $K_{p} \leq 3$ ), can be considered as a geomagnetically quiet time period. The characteristic time of the neutral composition recovery after a storm impulse event ranges from 7 to $12 \mathrm{~h}$, on average (Hedin, 1987), while it may require days for all altitudes down to $120 \mathrm{~km}$ in the atmosphere to recover completely back to the undisturbed state of the atmosphere (Richmond and Lu, 2000). We assume that the conditions are quiet if $K_{p} \leq 3$ during the $24 \mathrm{~h}$ before the measurement, otherwise the conditions are disturbed. The calculated average values of $\Psi_{M}$ over 3-month intervals are presented in Table 1 (columns III). It follows from Table 1 that the seasonal dependence of the average values of $\Psi_{M}$ is more strongly pronounced for the quiet conditions as compared with that for the disturbed conditions. When the NETEs observed under quiet conditions are ignored, the seasonal variations in $\Psi_{M}$ are approximately symmetric for the spring and autumn months. Thus, the asymmetry between spring and autumn NETE occurrences is produced by the asymmetry in the magnitudes of the spring and autumn sources of NETEs during geomagnetically quiet periods. During geomagnetically disturbed periods, a magnitude of spring and autumn sources of NETEs is approximately the same, and it leads to the symmetry between spring and autumn NETE occurrences. It is quite possible that there are differences in physical processes that form NETEs during time intervals with $K_{p} \leq 3$ and $K_{p}>3$ and these differences result in the asymmetry found between the spring and autumn $\Psi_{M}$ for quiet conditions.

As a result of our study, the asymmetry between spring and autumn NETE occurrences is found and the predominance of NETE occurrences in the winter and autumn months is discovered for geomagnetically quiet conditions. Comparable values of the average probabilities of the existence of NETEs during the winter and autumn months and the asymmetry between spring and autumn NETE occurrences found argue against the idea by Evans (1967) that the heating of thermal electrons at F-region altitudes is only caused by a simple re- lationship of these anomalous temperature enhancements to conjugate photoelectrons.

If we define the quiet conditions as the conditions, when $K_{p} \leq 3$ during $\delta t=24 \mathrm{~h}$ before the measurement, and otherwise the conditions are considered as disturbed, the number of the studied quiet and disturbed NETEs are $N u m_{q}=112$ and $N u m_{d}=280$, respectively. For $\delta t=12 \mathrm{~h}$ and $\delta t=0 \mathrm{~h}$ we obtain $\operatorname{Num}_{q}=140$, Num $_{d}=252$ and $N u m_{q}=207$, $\mathrm{Num}_{d}=185$, respectively. As $\delta t$ decreases, the number of events corresponding to quiet conditions, $\mathrm{Num}_{q}$, increases, while the seasonal NETE probability distribution is not changed within the errors (see Table 1, columns I and II). This result is consistent with the conclusion of our study, presented in Sect. 3.3, that the probability of observing a NETE depends on the current value of index $K_{p}$ rather than its previous values.

\subsection{Solar activity variations in the NETE occurrence prob- ability}

The dependency of the NETE occurrence probability $\Psi_{\mathrm{F} 10.7}$ on a daily solar activity index F10.7 is shown in Fig. 2. It can be seen from Fig. 2 that the value of $\Psi_{\mathrm{F} 10.7}$ is decreased with the solar activity index increase, and the dependence of $\Psi_{\mathrm{F} 10.7}$ on F10.7 within the accuracy of the analysis is very close to the linear one. Garner et al. (1994) had not enough data to draw the plot for the dependence of the NETE occurrence on F10.7. They only found that the NETE occurrence frequency is $26 \%$ per night for solar minimum conditions (F10.7 $\leq 100)$, 26\% per night for solar medium conditions $(100<\mathrm{F} 10.7 \leq 150)$, and $11 \%$ per night for solar maximum conditions $(\mathrm{F} 10.7>150)$. Our results do not agree with the conclusions of Garner et al. (1994) that the NETE occurrence frequency is the same for solar minimum and solar medium conditions, and that the solar maximum NETE occurrence frequency is noticeably lower than that at solar minimum or at solar middle activity levels. Our averaged values of $\Psi_{\mathrm{F} 10.7}$ are found to be $7.5 \%$ per hour for F10.7 $\leq 100$, $6.4 \%$ per hour for $100<\mathrm{F} 10.7 \leq 150$, and $5.0 \%$ per hour for F10.7 $>150$. The disagreement between our calculations and the results of Garner et al. (1994) may result from statistical 
fluctuations of the estimates of the occurrence probability, inasmuch as the fluctuations can be rather large for such a small data set which was used by Garner et al. (1994).

3.3 Geomagnetic activity variations in the NETE occurrence probability

Figure 3 shows the dependence of the NETE occurrence probabilities, $\Psi_{K_{p}}$ and $\Psi_{D_{s t}}$, on a 3-h geomagnetic index $K_{p}$ and on a 1-h index $D_{s t}$, respectively. Our calculations present a first evidence in the increase of $\Psi_{K_{p}}$ and $\Psi_{D_{s t}}$ with the geomagnetic activity. A small decrease in the last bin of the histogram for $\Psi_{K_{p}}$ is within the experimental error. It should be noted that Garner et al. (1994) were not capable of producing an evidence in favour of or against any correlation between geomagnetic activity and NETEs, because their data set was obtained predominantly under geomagnetically quiet conditions.

To find the time scales relevant for NETE formation as a response to geomagnetic activity, we calculated NETE's probability density functions depending not only upon the current value of the geomagnetic indices $K_{p}$ and $D_{s t}$, but on their previous values as well. The results obtained are shown in Fig. 4. It was found that the tendency for increasing the probability together with the geomagnetic activity persists up to $9 \mathrm{~h}$, for larger delays (12-15 h and more) the probability does not depend on $K_{p}$ or $D_{s t}$. However, the tendency is more pronounced for current values of $K_{p}$ or $D_{s t}$ rather than for delayed ones and becomes more weak with the delay increase, i.e. the NETE mechanism is associated with the current electrodynamic activity in the ionosphere-plasmasphere system. It is worth noting that, due to a relatively small number of events observed for large $K_{p}$, both current and previous, the errors for the two last bins in the top panel of Fig. 4 is higher than that for the other bins. In particular, the heights of black bins, which correspond to the results obtained with the indices measured $15 \mathrm{~h}$ before NETEs, are approximately the same within the errors.

To explain the tendency for the probability to increase together with the previous geomagnetic activity, we can suggest that NETEs are rather short-lived events, and their occurrence probability depend upon the current state of the ionosphere and plasmasphere rather than their previous state. The tendency may result from the fact that the typical durations of geomagnetic disturbances accompanied by NETEs are larger than $3 \mathrm{~h}$, may sometimes reach $12 \mathrm{~h}$, and only rare disturbances are longer.

\subsection{Temporal variability of NETE}

In Fig. 5, the dependence of the probability $\Psi_{\mathrm{t} 0}$ for an NETE to begin during 1-hour time periods on a local NETE onset time $\Psi_{t 0}$ is presented. In agreement with earlier conclusions of Garner et al. (1994), we found that NETEs are most likely to begin between 19:00 and 20:00 UT. Our calculations produce evidence in favour of the conclusion that the

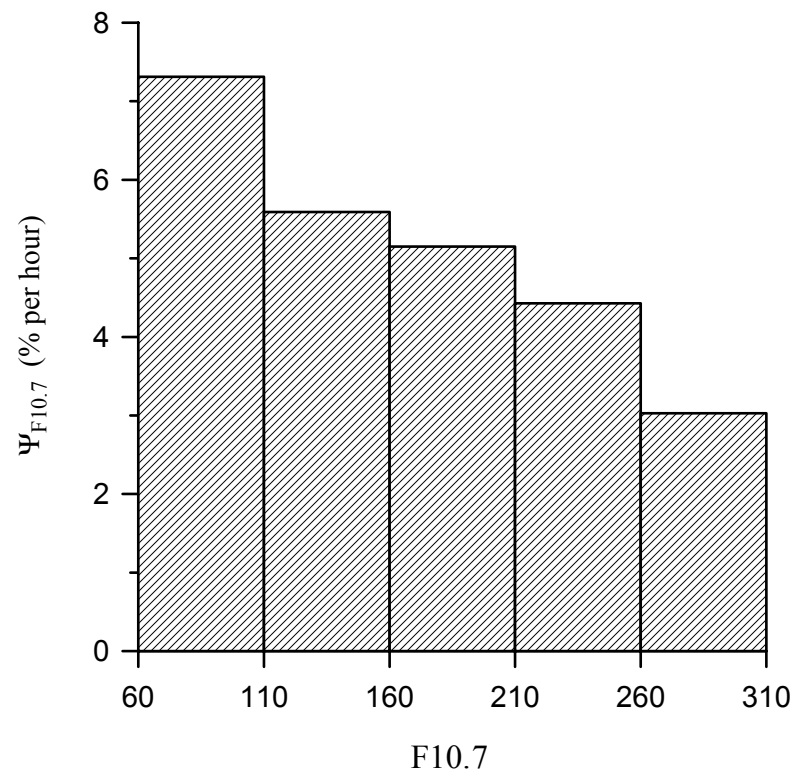

Fig. 2. The dependencies of the NETE occurrence probability $\Psi_{\mathrm{F} 10.7}$ on a daily solar activity index F10.7.

probability of the NETE onset is similar between 20:00 UT and 01:00 UT.

To study the distribution of NETE durations, we calculate a number, $S_{\Delta t 1 \leq \Delta t<\Delta t 2}$, of NETEs with durations within a given range, $\Delta t 1 \leq \Delta t<\Delta t 2$. The percentage, $P_{\Delta t}$, is defined as the ratio of $S_{\Delta t 1 \leq \Delta t<\Delta t 2}$ to the total number of NETE events. Figure 6 shows the statistical distribution of NETE durations. The distribution falls off exponentially,

$\log _{10}\left(\mathrm{P}_{\Delta \mathrm{t}}\right)=\mathrm{a}+\mathrm{b}\left(\mathrm{t}_{\mathrm{P}}-\mathrm{t}_{\mathrm{O}}\right)$,

where $a=2.357 \pm 0.013, b=-0.373 \pm 0.008$, the unit of $P_{\Delta t}$ is percent, and the unit of $t_{P}-t_{O}$ is an hour. It follows from our calculations that NETEs last between 1 and $3 \mathrm{~h}$ in most cases, with the percentage peak between 1 and $2 \mathrm{~h}$. This result does not support the conclusion by Garner et al. (1994) that the most probable duration of NETEs is from 2 to $3 \mathrm{~h}$.

\subsection{Amplitude variability of NETE}

To study the distribution of NETE amplitudes, we calcu-

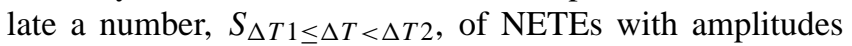
in a given range, $\Delta T 1 \leq \Delta T<\Delta T 2$. The amplitude of the studied NETEs are expressed in terms of the difference between the temperature at the peak, $T_{p}$, and the temperature, $T_{o}$, at the onset. The percentage, $P_{\Delta T}$, is defined as the ratio of $S_{\triangle T 1 \leq \Delta T<\Delta T 2}$ to the total number of NETE events. Figure 7 shows the statistical distribution of NETE amplitudes. The amplitude distribution has approximately an exponential distribution form: $\log _{10}\left(\mathrm{P}_{\Delta \mathrm{T}}\right)=a+b\left(T_{P}-T_{O}\right)$, where $a=1.648 \pm 0.009, b=(-8.03 \pm 0.12) \cdot 10^{-4}$, the unit of $\mathrm{P}_{\Delta \mathrm{T}}$ is percent, and the unit of $\mathrm{T}_{\mathrm{P}}-\mathrm{T}_{\mathrm{O}}$ is $\mathrm{K}$.

In agreement with the conclusions by Garner et al. (1994), the temperature increases are predominately between $100 \mathrm{~K}$ 

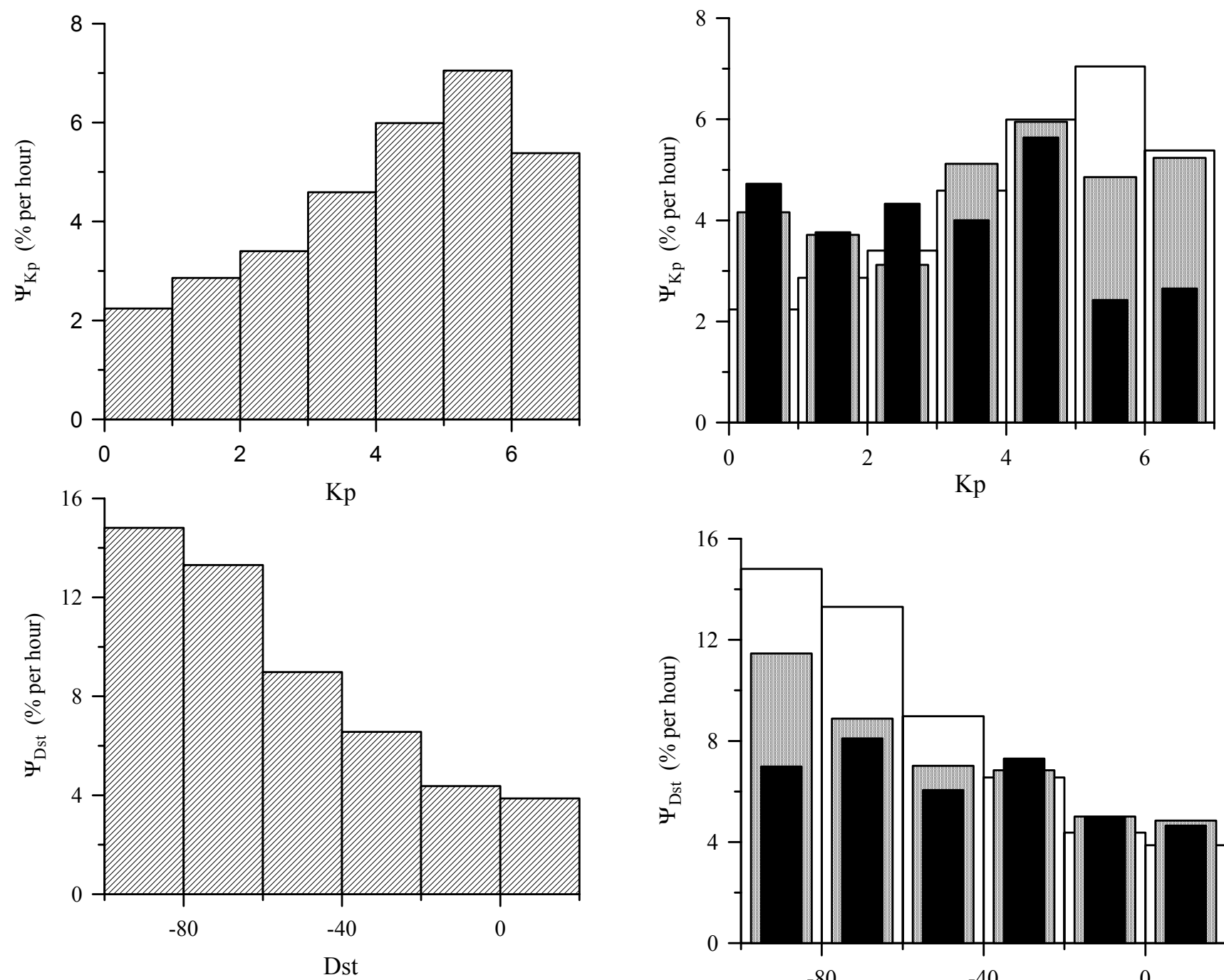

Fig. 3. The dependence of the NETE occurrence probability $\Psi_{K_{p}}$ and $\Psi_{D_{s t}}$ on a 3-h geomagnetic index $K_{p}$ (top panel) and 1-h index $D_{s t}$ (bottom panel).

and $300 \mathrm{~K}$. A maximum temperature increase of about $2600 \mathrm{~K}$ was observed. One can suggest that there exists a correlation between the NETE duration and amplitude. However, we did not find any relationship between the amplitude and duration of the NETEs studied.

\subsection{Relationship between the NETE amplitudes and geo- magnetic activity}

Figure 8 shows the relationship between the NETE amplitudes at $400 \mathrm{~km}$ and the geomagnetic activity measured by the index $K_{p}$ corresponding to the peak of the NETE. The amplitude of the studied NETEs are expressed in terms of the difference between the temperature at the peak, $T_{p}$, and the temperature, $T_{o}$, at the onset. It follows from Fig. 8 that there is a correlation between the quantities studied. The correlation coefficients of $\log _{10}\left(T_{p}-T_{o}\right)$ with $K_{p}$ and $D_{s t}$ are

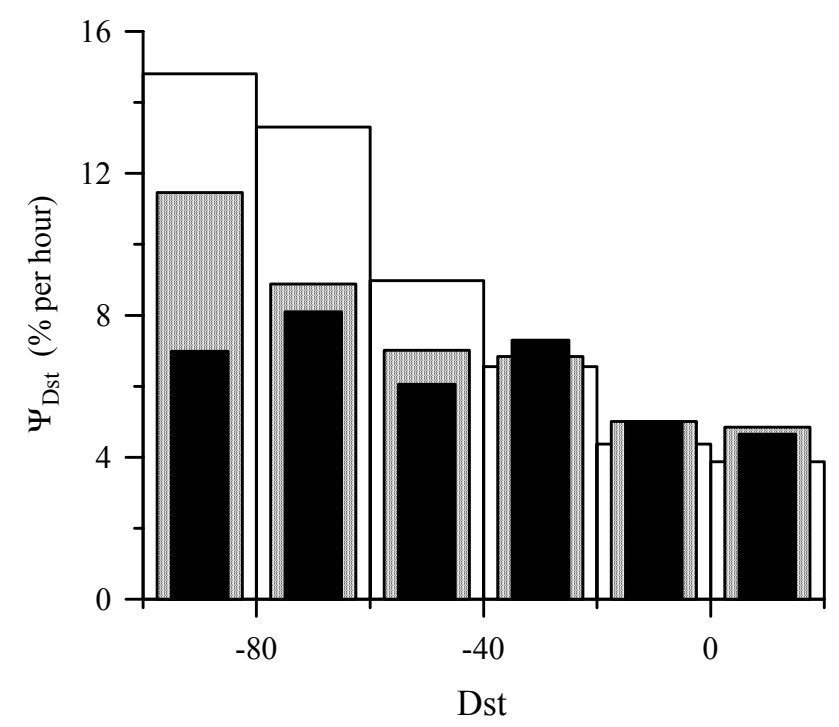

Fig. 4. The dependence of the NETE occurrence probability $\Psi_{K_{p}}$ and $\Psi_{D_{s t}}$ on current (white bins) and previous (gray and black bins) values of 3-h geomagnetic index $K_{p}$ (top panel) and 1-h index $D_{s t}$ (bottom panel). For both indices, the histograms with gray bins show the probability versus the indices measured $9 \mathrm{~h}$ before the NETEs peaks, while the black bins correspond to the results obtained with the indices measured $15 \mathrm{~h}$ before NETEs.

found to be $0.33 \pm 0.05$ and $-0.30 \pm 0.05$, respectively. We conclude that there exists a tendency for the NETE amplitude to increase together with the geomagnetic activity.

\subsection{Relationship between NETE and electron number den- sity changes}

The electron number density can rise or fall during a NETE. The relationship between the electron temperature and number density changes at $400 \mathrm{~km}$ during NETEs is shown in Fig. 9. The temperature changes are expressed in terms of the difference between the temperature at the peak and the temperature at the onset of NETEs. The electron number 


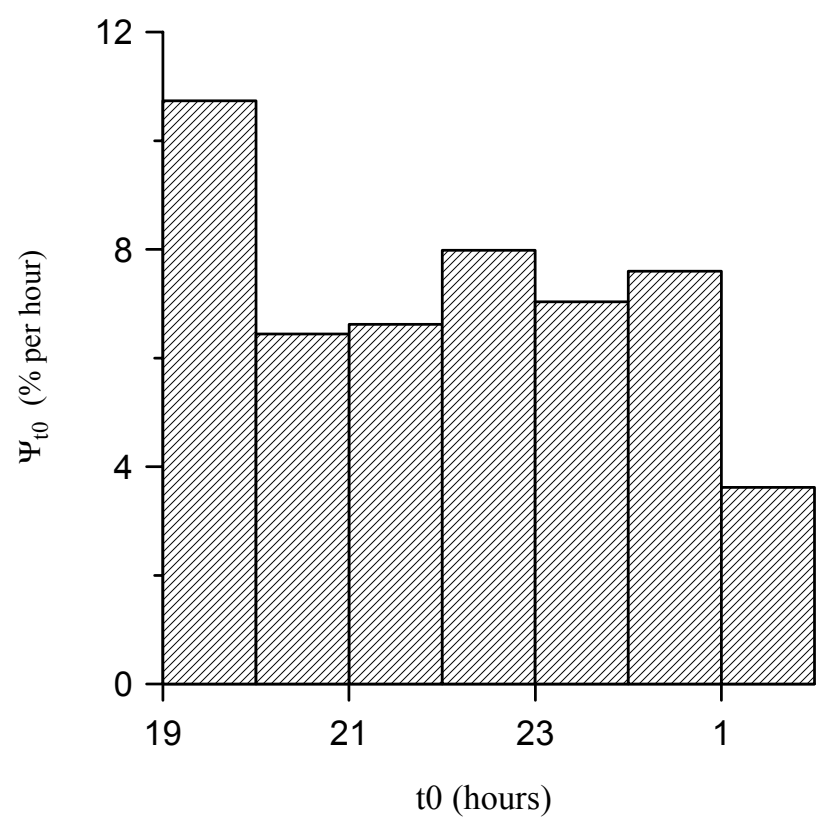

Fig. 5. The dependence of the NETE occurrence probability $\Psi_{t 0}$ for NETEs to begin during 1-hour time periods.

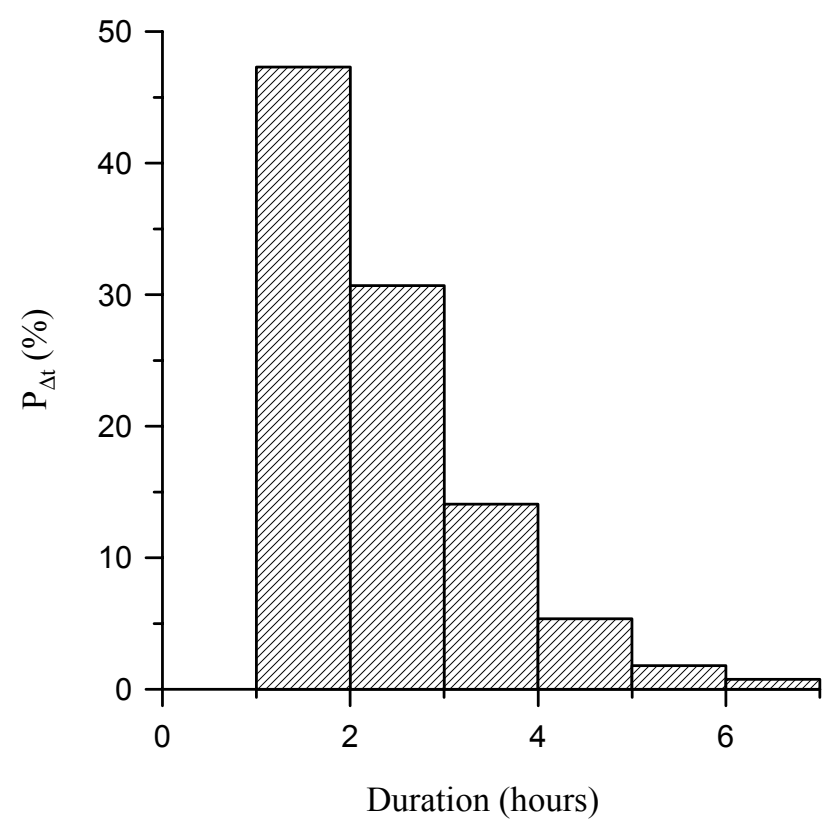

Fig. 6. The statistical distribution of NETE durations in 1-h bins.

density changes are the ratio of the electron number density, $N_{p}$, at the peak electron temperature to the electron number density, $N_{o}$, at the onset. In agreement with the previous results by Garner et al. (1994), during most of the NETEs the number density decreases and only a few events (about $4.5 \%$ in the data set used) are accompanied by the increase in density. From Fig. 9, it is easily seen that there exists an anti-correlation between the quantities studied, the correlation coefficient between $\left(N_{p}-N_{o}\right) / N_{o}$ and $\log _{10}\left(T_{p}-T_{o}\right)$

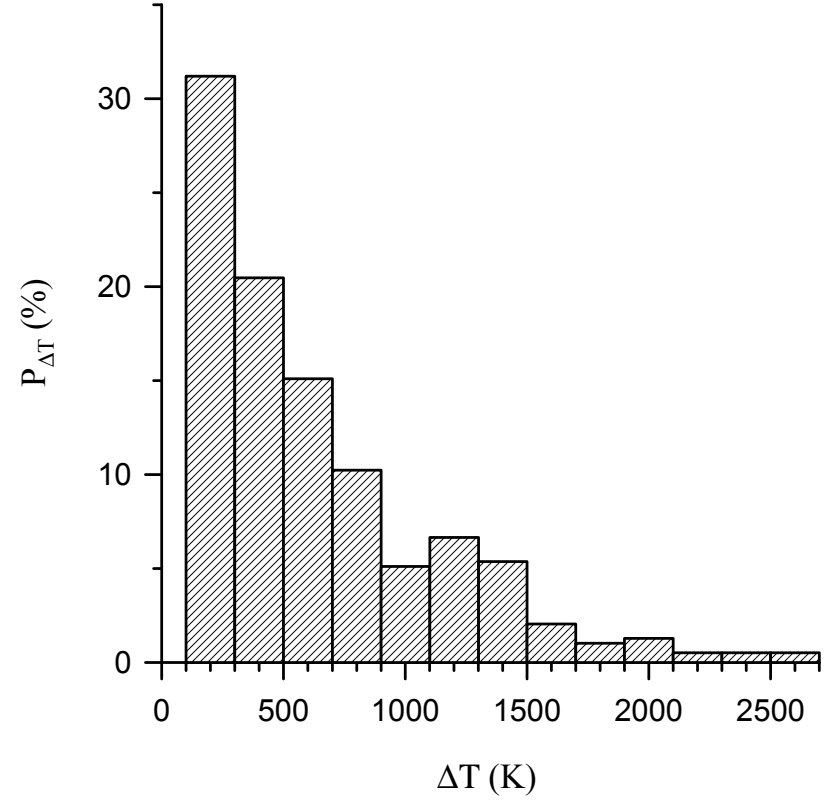

Fig. 7. The statistical distribution of NETE amplitudes in $200 \mathrm{~K}$ bins.

is $-0.47 \pm 0.04$. Thus, there exists a tendency for the plasma depression to increase as the NETE amplitude increases, and NEDEs are practically not observed during the NETEs. It is unclear in advance if the relationship between NETEs and number density changes at $400 \mathrm{~km}$ during NETEs is the same for quiet and disturbed geomagnetic conditions. We assume that the conditions are quiet if $K_{p} \leq 3$ during the $24 \mathrm{~h}$ before the measurement, otherwise the conditions are disturbed. We found that the correlation coefficient between $\log _{10}\left(T_{p}-T_{o}\right)$ and $\left(N_{p}-N_{o}\right) / N_{o}$ can be evaluated as $-0.46 \pm 0.05$ for quiet conditions and as $-0.44 \pm 0.06$ for disturbed conditions. We conclude that the correlation between the electron temperature and number density changes during NETEs is the same for quiet and disturbed geomagnetic conditions.

\subsection{Remarks on sources of the observed NETEs}

The physical origin of the observed NETEs is still unclear. The Millstone Hill's conjugate point is sunlit the whole night for the most of the winter period, but the NETEs only occur for a few hours. The study of Garner et al. (1994) and the results of this work show that the NETEs exist in the fall and spring months, i.e. these results do not support the idea of Evans (1967) that the heating of thermal electrons in the ionosphere is only caused by a simple relationship of these anomalous temperature enhancements to conjugate photoelectrons. It should be noted that Richards and Khazanov (1997) found that the plasmaspheric electron heating from photoelectrons must be doubled to reproduce the Millstone Hill observed topside electron temperature by the FLIP model of the ionosphere and plasmasphere during the periods 25-29 January 1993, i.e. the interaction between photoelec- 


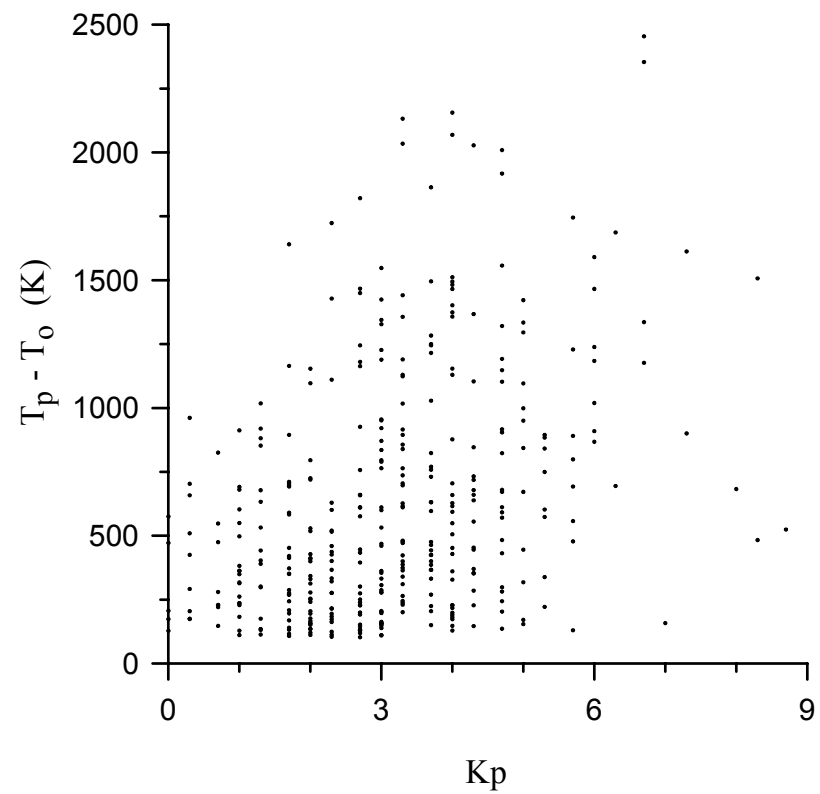

Fig. 8. The relationship between the measured electron temperature changes at $400 \mathrm{~km}$ during NETEs over Millstone Hill and a 3-h geomagnetic activity index $K_{p}$. The temperature changes are expressed as the difference between the temperature at the peak, $T_{p}$, and the temperature at the onset, $T_{o}$.

trons and thermal electrons cannot be considered as a source of an NETE if the current photoelectron theory is used. As a result, we believe that there is an additional heating rate, $Q_{a d}$, of thermal electrons in the plasmasphere.

The presence of photoelectrons in the ionosphere and plasmasphere is a potential source for the onset of plasma instabilities that can change the photoelectron distribution function and heating rate of electrons. The possible additional sources of the electron heating in the plasmasphere, such as wave-particle interactions, which can cause increased photoelectron scattering, and Coulomb collisions between ring current ions and plasmaspheric electrons and ions, could be the possible mechanisms to explain the observed electron temperature enhancements in the plasmasphere. Such a required additional heat source for electrons, over that for the usual photoelectron heating from Coulomb collisions, was used in many papers to make the modeled and measured electron temperatures agree (Horwitz et al., 1990; Pavlov, 1994, 1996, 1997; Balan et al., 1996; Richards and Khazanov, 1997; Pavlov et al., 2000, 2001). It is also possible that the physics of ionosphere-plasmasphere transport of photoelectrons is not well understood at this time.

As far as the authors know, there are no published reports of the comparison between the measured and modeled electron temperature in the ionosphere and plasmasphere along the same magnetic field line or close to the same magnetic field line except for the papers by Pavlov et al. (2000, 2001). As a result, there are no other published reports of an ionosphere-plasmasphere electron energy balance examination based on the electron temperature measurements in

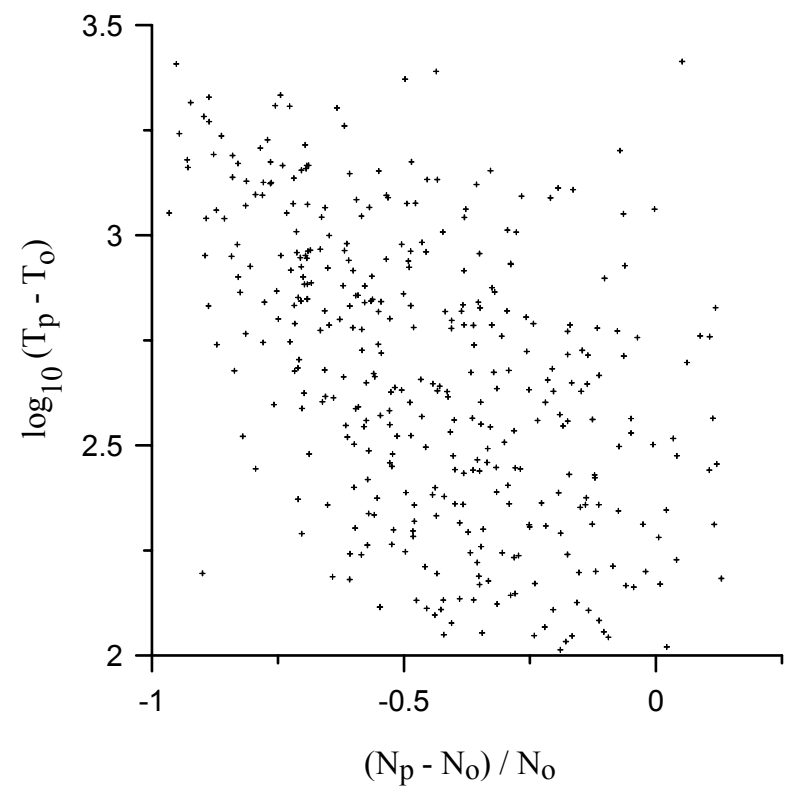

Fig. 9. The relationship between the measured electron temperature and number density changes at $400 \mathrm{~km}$ during NETEs over Millstone Hill. The temperature changes are expressed as the difference between the temperature at the peak, $T_{p}$, and the temperature at the onset, $T_{o}$. The electron number density changes are the ratio of the electron number density at the peak electron temperature, $N_{p}$, to the electron number density at the onset of the NETEs, $N_{o}$.

the ionosphere and plasmasphere close to the same magnetic field line. Several comparisons between the measured and modeled electron density and temperature obtained from the measurements by the Millstone Hill incoherent scatter radar in the ionosphere and the EXOS-D satellite in the plasmasphere for the undisturbed and geomagnetic storm periods of 20-30 January 1993 and 25-29 June 1990 were presented by Pavlov et al. (2000, 2001). Among other findings, it was found that additional heating brings the measured and modeled electron temperatures into agreement in the plasmasphere and into very large disagreement in the ionosphere if the electron heat flux along the magnetic field line is calculated with the use of classical formulas derived by Spitzer and Harm (1953). A new approach based on a new and effective nonlocal electron thermal conductivity coefficient, $\lambda_{e}$, along the magnetic field line was presented by Pavlov et al. $(2000,2001)$ to bring the measured and modeled electron temperatures into agreement in the ionosphere and plasmasphere. This approach leads to the electron heat flux, $F_{e}$, which is less than that given by the classical Spitzer and Harm (1953) theory of the electron thermal conductivity.

The observed NETE characteristics, described in Sects. 3.1-3.7, are determined by variations in $Q_{a d}, F_{e}$, and the cooling rates of thermal electrons. First of all, it should be noted that the NETEs are not regularly observed events. It means that the corresponding variations in $Q_{a d}$ are not regular as well. In a mixture of electrons and ions, the classical thermal conductivity coefficient depends only on 
$T$, while the thermal conductivity coefficient $\lambda_{e}$ is a function of $T,\left|\frac{\partial}{\partial s} T\right|$, and $N$ (Pavlov et al., 2000, 2001), where $S$ is the distance along the magnetic field line. As a result, the magnitude of $\left|F_{e}\right|$ is decreased under the action of the increase in $N$ or in $\left|\frac{\partial}{\partial s} T\right|$. In addition to the dependence of $\left|F_{e}\right|$ on $N$, we take into account that the electron-ion cooling rate of thermal electrons, which is the main cooling rate in the plasmasphere and topside ionosphere, is proportional to $N$ squared. As a result, there is the relationship between NETEs and electron number density changes described in Sect. 3.7. In spite of the fact that a dependence of $Q_{a d}$ on F10.7 is unknown, we can conclude that the increase in $N$ with the F10.7 increase can be part of the reason for the noted decrease in $\Psi_{\mathrm{F} 10.7}$ with the solar activity index increase (it is not inconceivable that it is the main reason).

We found that the NETE occurrence probability reveals the increase with geomagnetic activity characterized by the current geomagnetic activity indices $K_{p}$ or $D_{s t}$. It means that, at least partially, the dependence of the probability of the NETE occurrence on $K_{p}$ or $D_{s t}$ is determined by processes that control the increase of $Q_{a d}$ with the increase in $K_{p}$ or $\left|D_{s t}\right|$. The characteristic heating or cooling time of plasmaspheric thermal electrons is about one hour or less at L-shells which correspond to middle geomagnetic latitudes (Khazanov et al., 1992). Therefore, the NETE occurrence probability depends on the current state of the ionosphere and plasmasphere within this characteristic time, and does not depend on the previous state of the ionosphere and plasmasphere. The observed NETEs are rather short-lived events, with the typical characteristic time of about $3 \mathrm{~h}$ or less (see Sect. 3.4), and the dependence of the NETE occurrence probability on $K_{p}$ or $D_{s t}$ becomes more weak as we increase the delay between the NETE observation time and the $K_{p}$ or $D_{s t}$ determination time. It means that the typical characteristic time of $Q_{a d}$ is about $3 \mathrm{~h}$ or less as well. The increase of $Q_{a d}$ with geomagnetic activity is reasonable, but further work, which goes beyond the objective of our paper, is required to express this result in detailed changes in physical processes that determine the value of $Q_{a d}$. It should be noted as well that the main cooling rate in the topside ionosphere is proportional to $N$ squared, and, as a result, we assume that the variations in the electron density of the topside ionosphere with the $K_{p}$ or $\left|D_{s t}\right|$ increase can be part of the reason for the increase in the NETE occurrence probability found with the $K_{p}$ or $\left|D_{s t}\right|$ increase.

We assume that the seasonal variations found in the NETE occurrence probability and the temporal and amplitude variability found of the NETEs are determined (at least partially) by the corresponding variations in $Q_{a d}$. The theoretical study of the physical origins of these variations in $Q_{a d}$ goes beyond the objective of this work.

\section{Conclusions}

This paper is aimed at studying anomalous electron temperature enhancements observed on 336 nights over Millstone
Hill during Millstone Hill radar measurements on 730 nights from 1976 to 2001. The predominance of NETE occurrences in winter and autumn months is revealed. The found maximum value of the NETE probability is located in February and the NETE occurrence probability is the lowest in July. The asymmetry between spring and autumn NETE occurrences is found for NETEs that are observed during geomagnetically quiet time periods, when $K_{p} \leq 3$ during the $24 \mathrm{~h}$ before the measurements. The study of the seasonal dependence of the NETE probability was carried out with other definitions of the quiet conditions as well, i.e. the conditions were considered as quiet when $K_{p} \leq 3$ during the 12 and $0 \mathrm{~h}$ before the measurement, and otherwise considered as disturbed. The decrease in $\delta t$ leads to the increase in the number of quiet NETEs, while the seasonal NETE probability distribution is not changed within the errors. This result is consistent with the conclusion of our study, presented in Sect. 3.3, that the NETE occurrence probability depends upon the current level of geomagnetic activity rather than on previous levels.

The calculated value of the NETE occurrence probability is decreased with the solar activity index F10.7 increase, and this dependence is very close to the linear one. The NETE occurrence probability is increased, together with the geomagnetic activity (when the geomagnetic activity index $K_{p}$ increases and the index $D_{s t}$ decreases). The tendency is more pronounced for current values of $K_{p}$ or $D_{s t}$ rather than for delayed ones and becomes weaker with the delay increase. We found that the NETEs are most likely to begin between 19:00 and 20:00 UT, and the probability of the NETE onset is similar between 20:00 UT and 01:00 UT. The studied NETEs last between 1 and $3 \mathrm{~h}$ in most cases, with the percentage peak between 1 and $2 \mathrm{~h}$. The electron temperature increases are predominately between $100 \mathrm{~K}$ and $300 \mathrm{~K}$.

We did not find any relationship between the amplitude and duration of the NETEs studied. A correlation between $\log _{10}\left(T_{p}-T_{o}\right)$ and the $K_{p}$ and $D_{s t}$ geomagnetic activity indices is found, i.e. there is a tendency for the NETE amplitude to increase as the magnitude of $K_{p}$ or $\left|D_{s t}\right|$ increases.

We found that during most of the NETEs the number density decreases and only a few events (about $4.5 \%$ in the data set used) are accompanied by the increase in density. An anticorrelation between $\log _{10}\left(T_{p}-T_{o}\right)$ and $\left(N_{p}-N_{o}\right) / N_{o}$ is found. Thus, there exists a tendency for the plasma depression to increase as the NETE amplitude increases, and NEDEs are observed very rarely during NETEs. The correlation coefficient between the quantities studied is the same for quiet and disturbed geomagnetic conditions.

To determine whether there exists a difference between NETEs observed during different solar cycles, we chose the data subsets corresponding to 21 and 22 solar cycles, which are very similar in magnitude, and for each subset performed the statistical studies outlined above for the total set of data. It was found that, within the errors, the corresponding dependencies are the same for the cycles considered and for the entire data set. 
Acknowledgements. The research described in this publication was supported by grant 02-05-64204 from the Russian Foundation for Basic Research. Used electron temperatures and densities were observed by the incoherent scatter radar at Millstone Hill, Massachusetts. The authors would like to thank the staff of the Millstone Hill Observatory for a possibility to use the Madrigal Data Base. The authors would like to thank referees for their comments, which have assisted in improving the paper.

Topical Editor M. Lester thanks R. H. Comfort and S. Zhang for their help in evaluating this paper.

\section{References}

Babcock, Jr., R. R. and Evans, J. V.: Effects of geomagnetic disturbances on neutral winds and temperatures in the thermosphere observed over Millstone Hill, J. Geophys. Res., 84, 5349-5354, 1979.

Balan, N., Oyama, K.-I., Bailey, G. J., and Abe, T.: Plasmaspheric electron temperature profiles and the effects of photoelectron trapping and an equatorial high-altitude heat source, J. Geophys. Res., 101, 21 689-21 696, 1996.

Da Rosa, A. and Smith, F.: Behavior of the nighttime ionosphere, J. Geophys. Res., 72, 1829-1836, 1967.

Evans, J. V.: Cause of the midlatitude winter night increase in foF2, J. Geophys. Res., 70, 4331-4345, 1965.

Evans, J. V.: Mid-latitude F-region densities and temperatures at sunspot minimum, Planet. Space Sci., 15, 1387-1405, 1967.

Evans, J. V.: Millstone Hill Thomson scatter results for 1969, Lincoln Lab. Tech. Rep. 513, Lexington, MA, 1974.

Evans, J. V. and Holt, J. M.: Millstone Hill Thomson scatter results for 1972, Lincoln Lab. Tech. Rep. 530, Lexington, MA, 1978.

Garner, T. W., Richards, P. G., and Comfort, R. H.: Anomalous nighttime electron temperature events over Millstone Hill, J. Geophys. Res., 99, 11411-11 416, 1994.

Hedin, A. E.: MSIS-86 thermospheric model, J. Geophys. Res., 92, 4649-4662, 1987.

Horwitz, J. L., Comfort, R. H., Richards, P. G., Chandler, M. O., Chappel, C. R., Anderson, P., Hanson, W. B., and Brace, L. H.: Plasmasphere-ionosphere coupling. 2. Ion composition measurements at plasmaspheric and ionospheric altitudes and comparison with modeling results, J. Geophys. Res., 95, 7949-7959, 1990.

Khazanov, G. V., Nagy, A. F., Gombosi, T. I., Koen, M. A., and Cariglia, S. J.: Analytic description of the electron temperature behavior in the upper ionosphere and plasmashere, Geophys. Res. Lett., 19, 1915, 1992.

Pavlov, A. V.: The role of vibrationally excited nitrogen in the for- mation of the mid-latitude ionospheric storms, Ann. Geophysicae, 12, 554-564, 1994.

Pavlov, A. V.: Mechanisms of the electron density depletion in the SAR arc region, Ann. Geophysicae 14, 211-221, 1996.

Pavlov, A. V.: Subauroral red arcs as a conjugate phenomenon: comparison of OV1-10 satellite data with numerical calculations, Ann. Geophysicae, 15, 984-998, 1997.

Pavlov, A. V., Buonsanto, M. J., Schlesier, A. C., and Richards, P. G.: Comparison of models and data at Millstone Hill during the 5-11 June, 1991, storm, J. Atmosph. Terr. Phys., 61, 263-279, 1999.

Pavlov, A. V. and Oyama, K.-I.: The role of vibrationally excited nitrogen and oxygen in the ionosphere over Millstone Hill during 16-23 March 1990, Ann. Geophysicae, 18, 957-966, 2000.

Pavlov, A. V., Abe, T., and Oyama, K.-I.: Comparison of the measured and modeled electron densities and temperatures in the ionosphere and plasmasphere during 20-30 January 1993, Ann. Geophysicae, 18, 1257-1272, 2000.

Pavlov, A. V., Abe, T., and Oyama, K.-I.: Comparison of the measured and modeled electron densities and temperatures in the ionosphere and plasmasphere during the period of 25-29 June 1990, J. Atmosph. Terr. Phys., 63, 605-616, 2001.

Pavlov, A. V. and Foster, J. C.: Model/data comparison of F-region ionospheric perturbation over Millstone Hill during the severe geomagnetic storm of 15-16 July 2000, J. Geophys. Res., 105, 29 051-29070, 2001.

Rao, M. N., Raj, P. E., and Jogulu, C.: A study of the post-sunset increase in the F2-region electron density at low- and middle latitudes in the Asian zone during sunspot maximum and minimum periods, Ann. Geophysicae, 38, 357-365, 1982.

Richards, P. G., Buonsanto, M. J., Reinisch, B. W., Holt, J., Fennelly, J. A., Scali, J. L., Comfort, R. H., Germany, G. A., Spann, J., Brittnacher, M., and Fok, M.-C.: On the relative importance of convection and temperature to the behavior of the ionosphere in North America during January 6-12, 1997, J. Geophys. Res., 105, 12 763-12 776, 2000.

Richards, P. G. and Khazanov, G. W.: On the thermal electron energy balance in the ionosphere in January 1993 and June 1990, J. Geophys. Res., 102, 7369-7377, 1997.

Richmond, A. D. and Lu, G.: Upper-atmospheric effects of magnetic storms: a brief tutorial, J. Atmosph. Terr. Phys., 62, 11151127, 2000.

Santani, S. and Breig, E. L.: Winter nighttime ion temperatures and energetic electrons from OGO 6 plasma measurements, J. Geophys. Res., 86, 3595-3609, 1981.

Spitzer, L. and Harm, R.: Transport phenomena in a completely ionized gas, Phys. Rev. 89, 977-981, 1953. 